\title{
PRESSURE INDUCED STRUCTURAL TRANSFORMATION IN THULIUM MONOTELLURIDE
}

\section{S. Usha Devi and A.K. Singh}

Materials Science Division

National Aeronautical Laboratory

Bangalore 560017

India

(Received 4 May 1984; in revised form 30 July 1904 by A.R.verma)

\begin{abstract}
Thulium monotelluride undergoes a structural transformation at 15 Gga from NaCl-type structure to a hexagonal structure with unit cell dimensions: $a=4.07 \mathrm{~A}$ and $\mathrm{C}=6.84 \mathrm{~A}$. The high pressure diffraction pattern is explained reasonably well by anti-niAs-type structure. The volume change associated with the transformation is $\mathbf{3 . 8}$ per cent.
\end{abstract}

Thulium monotelluride, at one fatmosphere and room temuerature. has Hacl-type structure. The studies on manetic susceptibility and lattice parameter indicate that the $\mathrm{m}$ fon is in a divient state(1). The pressure dependences of electrical resistivity[2] and the unit cell wolume $(3,4)$ suggest that the Tmlan changes continuously to a tivalent state as the pressure is increased. The earlier work on the equation of state of TmTe was imited to a pressure up to $13 \mathrm{gpa}$. 15 gPa TmTe undergoes a structural tramsormation. The analysis of the crystal structure of the high pressure phase is reported here.

The specimens used in the present experiments were same as those wised in the earlier studies $\{3,4)$. A preliminary characterization of the specimen was done by $x$-ray analysis. The sharpness of the difiraction patterns taken with CuK $\alpha$ radiation indicated that the specimen was highly crystalline, and all the observed diffraction lines could be explained on the basis of a cubic phase (NaCl-type). The lattice paraneter (a $=6.354 \pm 0.002$ A) was obtained from the Nelson-Riley plot ising the high angle reflections. the present value of lattice paraheter agrees well with the value $(a=6.364$ A $=6$ reported by Bucher et al[5], and also with the value $(\mathrm{la}=6.35 \mathrm{~A})$ reported recently by haidis and Fitzler[6].

The $x$-ray experiments at eleva. ed pressures were doṇe with a Bjahond-anvil camera[7l. The specimen Hem finely powdered and placed betveen the anvils without gasket. The Wiitfraction patterns were recorded bith a cylindrical film cassette using MoKa radiation. To avoid the complication arising from the presence of the diffraction lines from the pressure marker TmTe specimen alone (without pressure marker) was used.. The $x$-ray patterns taken at about 15 gea showed a new set of lines, indicating a change in the crystal structure. The new set of lines could be indexed in the hexagonal system with the following lattice parameters: $a=4.07 \pm$ $0.04 \mathrm{~A}$ and $\mathrm{C}=6.84 \pm 0.07 \mathrm{~A}$. The diffraction pattern also contained lines from the untransformed NaClphase. The pressure on the specimen was estimated from the cell volume of the coexisting Nacl-phase of TmTe and the equation of state determined,earlier $(3,4)$.

The intensities of the diffraction lines were estimated visually using an intensity scale containing diffraction spots of graded optical densities. The measured intensities were corrected for Lofentz-polarization and multiplicity factors. The correction factor for the absorption in the anvil and the specimen (with an assumed thickness of 25 microns 1 did not vary significantly over the observed range of reflections. The abosiption correction factor was therefore treated as a scale factor. The maximum error in the intensities arising from the neglect of the absorption correction factor was about 10 percent. The overall uncertainty in the estimation of intensities is rather large (nearly 25 percent) because of the presence of slight preferred orientation in the high pressure diffraction patterns. The intensity of the strongest observed reflection was taken as 100 and the intensities of the other reflections scaled 
accordingly. The observed d-values and the intensities are listed in Table 1 . of the reflections with large calculated intensities were not observed experimentally. Further, even for

Table 1. Crystallographic data on the high pressure phase of TmTe (hexagonal, $a=4.07 \mathrm{~A}$ and $\mathrm{C}=6.84 \mathrm{~A})$ at $15 \mathrm{GPa}$.

\begin{tabular}{|c|c|c|c|c|}
\hline$a_{o b s}(A)$ & $d_{\text {cal }}(A)$ & $\mathrm{hkl}$ & Iobs & $I_{c a l}$ \\
\hline & 3.525 & 100 & $0^{+}$ & 5 \\
\hline - & 3.419 & 002 & 0 & 2 \\
\hline 3.054 & 3.133 & 101 & 100 & 100 \\
\hline 2.926 & $2.945^{\star}$ & $200 *$ & & \\
\hline 2.454 & 2.454 & 102 & 95 & 110 \\
\hline 2.093 & $\begin{array}{l}2.035 \\
2.082^{\star}\end{array}$ & $\begin{array}{l}110 \\
220^{*}\end{array}$ & 71 & 64 \\
\hline 1.901 & 1.914 & 103 & 16 & 27 \\
\hline - & 1.762 & 200 & 0 & 0.8 \\
\hline - & 1.749 & 112 & 0 & 2 \\
\hline 1.715 & $\begin{array}{l}1.707, \quad 710 \\
1.700 *\end{array}$ & $\begin{array}{l}201,004 \\
222 *\end{array}$ & 42 & 20,13 \\
\hline 11.563 & 1.567 & 202 & 29 & 30 \\
\hline - & 1.538 & 104 & 0 & 0.9 \\
\hline 1.420 & 1.394 & 203 & 35 & 10 \\
\hline - & 1.332 & 210 & 0 & 0.6 \\
\hline 1.306 & $\begin{array}{l}1.309, \\
1.317^{*}\end{array} \quad 308$ & $\begin{array}{l}114,211 \\
420^{*}\end{array}$ & 37 & 34,17 \\
\hline
\end{tabular}

* - NaCl phase

+ The lowest measurable intensity is 1.0 .

The number, $n$, of the molecules in the unit cell is taken as 2, because it gives a reasonable value ( $-3.8 \%)$ for the volume change., $\Delta(t)$, associated with the transformation. The choice of any other value of $n$ leads to inadmissible value of $\Delta V(t) . \quad A$ search was made in the literature for the possible hexagonal structures for compounds of type Rx, containing two; molecules in the unit cell. The possible structures are zno-type, NiAs-type. and anti-NiAs-type. First, the zno-type structure was tried. With the Tm-ions in Zn-positions 'and Te-ions in the 0-positions, the intensities of the possible reflections with the interplanar spacings up to 1.3 A were calculated. The calculated intensity corresponding to the strongest observed reflection was taken as 100 and the intensities of the other reflections were scaled accordingly. A comparison of the calculated intensities with the observed values indicated that some the observed reflections the discrepancies between the observed and calculated intensities were serious. The Niswtype structure was tried next, and was also discarded in view of the serious discrepancies. between the observed and the calculated intensities. The anti-NiAs-' type structure with Tm-ions (R-ions) at $(1 / 3,2 / 3,1 / 41$ and $(2 / 3,1 / 3$, $3 / 41$, and $\mathrm{Te}-$ ions $(\mathrm{X}-\mathrm{ions})$ at $(0$, $0,0)$ and $(0,0,1 / 2)$ was tried next. The observed and the calculated intensities are listed in Table 1. Out of the three structures tried, the anti-NiAs structure explains the high pressure intensity. data best. Rather large discrepancies in the case of 103 and 203: reflections seem to arise from the preferred orientation effects.

The atomic arrangement in the high pressure phase of TmTe is shown in Fig, 1. Each Tm-ion, marked ? in Fig. 1, is srrounded by six $\mathrm{Te}$. ions (1-6) forming a trigonal prism: 


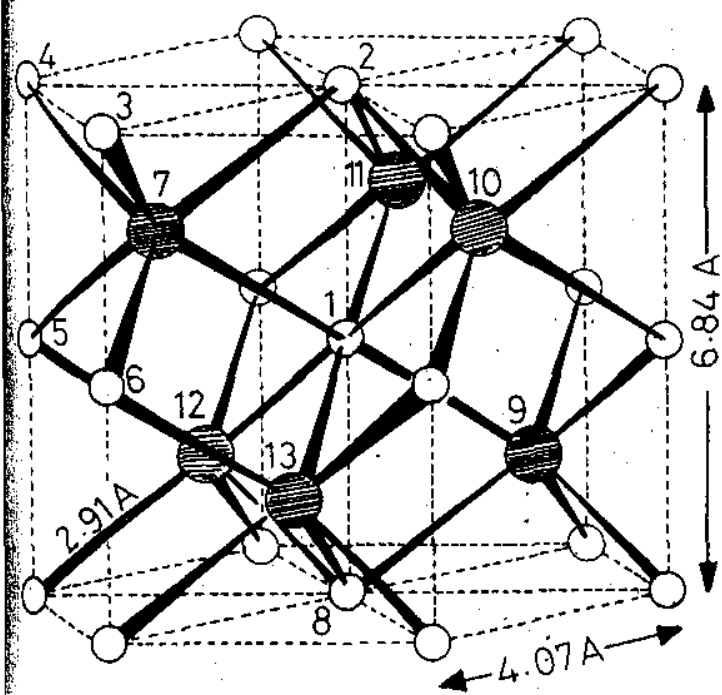

O Te

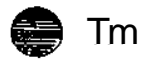

rig.1. The atomic arrangement in high pressure ohise of TmTe at is Gga.

he distance between the $\mathrm{Tm}-$ and te-ion being 2.91A at is GPa. Each (t)-ion (1) has six Tm-ions (9-13 (ind at a distance of 2,91 A at 15.GPa; the six Tm-ions occupy octadedral positions. The environment of the Te-ion in the high pressure phase is the same as in the low pressure phase (Macl-type structure). However, in the high pressure phase of TmTe, each Te-ion (1) has also two Te-ions $(2$ and 8 ) at a distance of 3.42 A. Since the nearest Te-Te distance is nearly 18 percent larger than the nearest Te-Tm distance, only a weak bonding can exist between the nearest Te-ions. Thus, the transformation results in a rearrangement of the surroundings of the Tm-ions, leaving the arrangement of ions around the Te-ions practically unaltered.

The other compounds, in the group of rare earth monochalcogenides, which exhibit structural transformations $(3,4,8)$ under pressure are PrTe, SmTe, EuTe, Euse and Euo. At one atmosphere these compounds, like TmTe, have Nacl-type structure and are found to transform under pressure to cscl-type structure. The high pressure phase of TmTe however is hexagonal. In this respect TmTe differs from the other rare earth monochalcogenides. The possibility that TmTe transforms to cscl-type structure, in conformity with the behaviour of other rare earth monochalcogenides, at still higher pressures is not ruled out.

\section{REFERENCES}

E.Bucher, A.C.Gossard, K. Andres, J.P.Mita 6 A.S.Cooper, Proc. Eighth Rare Earth Research Conference, Editors: T.H.Henrie $\quad$ R.E.Lindstrom, vol. 1, p74 (1970). A. Jayaraman, E.Bucher 6 D.B. Mowhan, Proc. Eighth Rare Earth Research Conference, Editors: T.H.Henrie and R,E,Lindstrom vol. i, p 333(1970).

A. Chatterjee, A.K.Singh 6 A.Jayaraman, Phys. Rev. B6, 2285 (1972). A.Jayaraman, A.K.Singh \& A.Cha- tterjee $\&$ S. Usha Devi, Phys. Rev. B9, 2514 (1974).

5. E.Bucher, K.Andres, F, J,Disalvo, J.P. Maita, A.C.Gossard, S.Coopen \& G.W.HLl JI. Phys. Rev. Bll, $500(9975)$.

6. E.Kaldis \& B.Fritzler, Progress. Solid State Chem. 14, 95 (1982).

7. W.A.Bassett, T.Takahashi \& P.W. Stook, Rev. Sci. Instrum, 38, 37 (1967).

8. A,X.Singh, A.Jayaraman \& A.Chatterjee, Solid State. Commun. $9,1459(1971)$. 